\title{
Video Article \\ Quantitative Analysis of Synaptic Vesicle Pool Replenishment in Cultured Cerebellar Granule Neurons using FM Dyes
}

\author{
Giselle Cheung ${ }^{1}$, Michael A. Cousin ${ }^{1}$ \\ ${ }^{1}$ Membrane Biology Group, Centre for integrative Physiology, University of Edinburgh \\ Correspondence to: Michael A. Cousin at M.Cousin@ed.ac.uk \\ URL: https://www.jove.com/video/3143 \\ DOI: doi:10.3791/3143
}

Keywords: Neuroscience, Issue 57, synaptic vesicle, neuron, recycling pool, readily releasable pool, reserve pool, replenishment, FM dyes, exocytosis, endocytosis

Date Published: $11 / 11 / 2011$

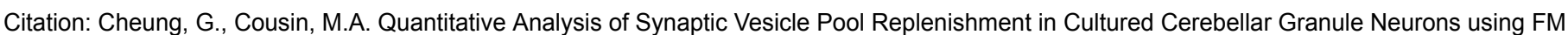
Dyes. J. Vis. Exp. (57), e3143, doi:10.3791/3143 (2011).

\section{Abstract}

\begin{abstract}
After neurotransmitter release in central nerve terminals, SVs are rapidly retrieved by endocytosis. Retrieved SVs are then refilled with neurotransmitter and rejoin the recycling pool, defined as SVs that are available for exocytosis ${ }^{1,2}$. The recycling pool can generally be subdivided into two distinct pools - the readily releasable pool (RRP) and the reserve pool (RP). As their names imply, the RRP consists of SVs that are immediately available for fusion while RP SVs are released only during intense stimulation ${ }^{1,2}$. It is important to have a reliable assay that reports the differential replenishment of these SV pools in order to understand 1) how SVs traffic after different modes of endocytosis (such as clathrindependent endocytosis and activity-dependent bulk endocytosis) and 2) the mechanisms controlling the mobilisation of both the RRP and RP in response to different stimuli.
\end{abstract}

FM dyes are routinely employed to quantitatively report SV turnover in central nerve terminals ${ }^{3-8}$. They have a hydrophobic hydrocarbon tail that allows reversible partitioning in the lipid bilayer, and a hydrophilic head group that blocks passage across membranes. The dyes have little fluorescence in aqueous solution, but their quantum yield increases dramatically when partitioned in membrane ${ }^{9}$. Thus FM dyes are ideal fluorescent probes for tracking actively recycling SVs. The standard protocol for use of FM dye is as follows. First they are applied to neurons and are taken up during endocytosis (Figure 1). After non-internalised dye is washed away from the plasma membrane, recycled SVs redistribute within the recycling pool. These SVs are then depleted using unloading stimuli (Figure 1). Since FM dye labelling of SVs is quantal ${ }^{10}$, the resulting fluorescence drop is proportional to the amount of vesicles released. Thus, the recycling and fusion of SVs generated from the previous round of endocytosis can be reliably quantified.

Here, we present a protocol that has been modified to obtain two additional elements of information. Firstly, sequential unloading stimuli are used to differentially unload the RRP and the RP, to allow quantification of the replenishment of specific SV pools. Secondly, each nerve terminal undergoes the protocol twice. Thus, the response of the same nerve terminal at $\mathrm{S} 1$ can be compared against the presence of a test substance at phase S2 (Figure 2), providing an internal control. This is important, since the extent of SV recycling across different nerve terminals is highly variable $^{11}$

Any adherent primary neuronal cultures may be used for this protocol, however the plating density, solutions and stimulation conditions are optimised for cerebellar granule neurons $(\mathrm{CGNs})^{12,13}$.

\section{Video Link}

The video component of this article can be found at https://www.jove.com/video/3143/

\section{Cerebellar Granule Neuron Preparation}

1. Autoclave approximately $10025 \mathrm{~mm}$ diameter coverslips (Table 1).

2. Place coverslips in a $50 \mathrm{ml}$ sterile tube containing sterile poly-D-lysine solution (Table 2). Place on a rotating platform for $2 \mathrm{~h}$ to coat the coverslips.

3. Dry coated coverslips on sterile tissue paper in a laminar flow hood (Table 1).

4. Place coverslips into sterile 6-well plates and warm in a $\mathrm{CO}_{2}$ incubator (Table 1). Coverslips can be stored for 1 month at $4^{\circ} \mathrm{C}$ for before use.

5. Euthanize a 7 day old Sprague Dawley rat pup according to local ethical committee guidelines. We euthanized pups using cervical dislocation.

6. Dissect the cerebellum and place it in a sterile petri dish containing a phosphate buffered salts solution (solution B, Table 3).

7. Repeat steps 1.5 and 1.6 for $4-6$ rat pups.

8. Cerebella are then placed on the sterile stage of a Mcllwain Tissue Chopper (Table 1). Tissue is chopped at $375 \mu \mathrm{m}$ intervals before rotating the stage through $90^{\circ}$ and repeating the process. 
9. The chopped cerebella are transferred into a trypsin solution (solution $\mathrm{T}$, Table 4 ) which had previously been warmed to $37^{\circ} \mathrm{C}$.

10. Incubate cerebella at $37^{\circ} \mathrm{C}$ for 20 min with gentle agitation approximately every $5 \mathrm{~min}$.

11. During tryptic digestion, flame polish three sterile glass pipettes (Table 1) using a Bunsen flame. Use the flame to create a fine bore, a medium bore and a wide bore at the respective mouths of the pipettes.

12. After 20 min incubation in solution T, add $20 \mathrm{ml}$ of a trypsin / DNase inhibitor solution (solution W, Table 5) to the cerebellar suspension and pellet cells at $1,000 \mathrm{~g}$ for $1 \mathrm{~min}$ in a benchtop centrifuge (Table 1 ).

13. Decant the supernatant and resuspend the cell pellet in $1.5 \mathrm{ml}$ of a concentrated trypsin / DNase inhibitor (solution $\mathrm{C}$, Table 6 ) using the widest bore pipette.

14. Triturate the cells using first the wide bore pipette, then the medium bore and finally the narrow bore until the cell suspension is homogenous. This is a key step, the suspension must be homogenous at this stage.

15. Layer the cell suspension on top of $10 \mathrm{ml}$ of a prewarmed $\left(37^{\circ} \mathrm{C}\right)$ bovine serum albumin supplemented Earles Balanced Salts Solution (Table 7) in a sterile $15 \mathrm{ml}$ tube.

16. Centrifuge the suspension for $5 \mathrm{~min}$ at $1,500 \mathrm{~g}$ and resuspend the cell pellet in $2 \mathrm{mls}$ of prewarmed $\left(37^{\circ} \mathrm{C}\right)$ culture medium (Table 8$)$.

17. Estimate the cell number using a haemocytometer (Table 1) and dilute the cell suspension to a final density of $3.3 \times 10^{6}$ cells per ml.

18. Cells are plated by adding $75 \mu$ of the cell suspension to the centre of poly-D-lysine-coated coverslips (final density $2.5 \times 10^{5}$ ).

19. The culture plates containing the coverslips are placed in the $\mathrm{CO}_{2}$ incubator for 60 min to allow the cells to adhere.

20. Add $1.5 \mathrm{ml}$ of culture medium into each well taking care not to disturb the plated cells and return the culture plates to the $\mathrm{CO}_{2}$ incubator.

21. The following day replace the culture medium with fresh culture medium supplemented with the mitotic inhibitor cytosine arabinoside (Table 8). This arrests proliferation of glial cells in culture.

\section{Experimental Setup}

1. Basic experimental setup should consist of the following (see Tables 1 and 9 for specific equipment and software used):

- Inverted epi-fluorescence microscope

- Cooled CCD camera

- Fluorescent light source (monochromator or filter wheel)

- Gravity perfusion apparatus

- Imaging chamber with parallel platinum electrodes

- Electrical stimulator

- Computer

- Image acquisition software

2. Experiments should be performed in the dark or under red light conditions with minimum fluorescent illumination of the sample to avoid FM dye bleaching.

3. Experiments are carried out at room temperature. If physiological temperature is required, a temperature-controlled perfusion system may be used.

\section{Sample Preparation}

1. Cultures should be used after 8-12 days in vitro.

2. Transfer a single coverslip to saline solution (Table 10) for $10 \mathrm{~min}$ at room temperature to allow stabilisation in new medium.

3. Remove the coverslip, dry its underside and the area surrounding the attached cells with a small piece of paper towel or absorbent paper.

4. Using silicone grease (Table 2), glue the coverslip to the underside of the imaging chamber. Cells should be between the two parallel wires. Sufficient silicone grease should be used to completely seal the chamber but without any grease entering the center of the bath chamber.

5. Gently fill the bath chamber with $\sim 260 \mu$ saline solution and then fill the input tubing with the same solution.

6. Glue a clean coverslip with silicone grease to the top of the chamber to seal it. The input and output tubing can be used to remove any air bubbles trapped in the chamber. It is important that the electric circuit is not interrupted by air bubbles.

7. Immobilise the imaging chamber in a stainless steel platform and check for leaks by gently perfusing saline solution through the input tubing

8. Mount the assembled chamber on the stage of an inverted microscope, and connect the chamber to a gravity perfusion system, having first primed the inlet with saline solution.

9. Attach connecting wires of the chamber to the electrical stimulator.

10. Add a drop of immersion oil to the objective if an oil lens is used. Focus on the cells in the middle of the chamber using bright field illumination.

\section{S1 Phase}

1. Perfuse neurons with $1.5 \mathrm{ml}$ of FM dye (Table 2 ) diluted in saline solution.

2. Stimulate neurons to evoke dye uptake using the attached stimulator.

3. After stimulation, perfuse neurons with fresh saline solution for 2 min to wash away excess FM dye (flow rate $7 \mathrm{ml} / \mathrm{min}$ ). Glial contamination in the CGN culture system is less than $5 \%{ }^{14}$, therefore this time frame is sufficient to remove dye.

4. Leave neurons to rest for $8 \mathrm{~min}$.

5. During this interval, locate axonal networks where individual FM dye-loaded nerve terminals are visible using fluorescein wavelengths (excitation, $480 \mathrm{~nm}$; emission, >550 nm). Avoid areas with clusters of cells. Keep illumination to a minimum at this step, since intense excitation can result in dye phototoxicity. Obvious signs of this are blebbing of axons and a lack of dye unloading (due to dye fixing).

6. Re-focus image immediately before image acquisition since a slight drift may have occurred during the rest period.

7. Begin time-lapse image acquisition at the rate of 1 frame every $4 \mathrm{~s}$.

8. After acquiring $5-10$ baseline images, evoke exocytosis of the RRP by delivering a $30 \mathrm{~Hz}$ stimulation for $2 \mathrm{~s}(60 \text { action potentials })^{8}$. Commence stimulation manually immediately after frame capture. 
9. After acquiring another 10 images, evoke SV exocytosis of the RP using three stimulations of $40 \mathrm{~Hz}$ for $10 \mathrm{~s}$ ( 400 action potentials), each 30 s apart ${ }^{8}$.

10. Acquire another 5 - 10 images and then pause image acquisition.

\section{Recovery Phase (see Figure 2)}

1. Allow neurons to recover over at least $20 \mathrm{~min}$.

2. Optional - If the effect of a drug on endocytosis is to be tested, perfuse neurons with drug solution during this period (Figure $2 \mathrm{~b})^{3,8}$.

\section{S2 Phase}

1. Repeat $\mathrm{S} 1$ phase protocol (section 4) for a control experiment using the same field of view as in S1.

2. Optional - If the effect of a drug on endocytosis is to be tested, perfuse neurons with the drug solution supplemented with FM dye (Figure $2 \mathrm{~b})^{3,8}$.

3. Optional - Alternatively if drug effects on exocytosis are of interest, perfuse neurons with the drug solution both before and during the RRP and RP unloading stimuli (Figure $2 \mathrm{c})^{3}$.

\section{Data Analysis}

1. Use ImageJ and Microsoft Excel or similar software for data analysis.

2. For analysis, an image sequence in stack format is required. Some imaging software may export sequences as single images. If this is the case, convert images to a stack using an ImageJ built-in function Image>Stacks $>$ Images to stack.

3. Adjust the brightness and contrast of the stack to maximize the dynamic range. Image $>$ Adjust $>$ Brightness/Contrast (Figure 3a).

4. If significant horizontal drift has occurred during the experiment, run StackReg (http://bigwww.epfl.ch/thevenaz/stackreg/) and TurboReg (http://bigwww.epfl.ch/thevenaz/turboreg/) plugins on ImageJ to align image stack (Figure 3b).

5. Run Time Series Analyzer plugin (http://rsbweb.nih.gov/ij/plugins/time-series.html) (Figure 3c).

6. Define regions of interest (ROIs) over at least 90 nerve terminals. These should be identical (circular ROIs with $1.5 \mu \mathrm{m}$ diameter) It is helpful to toggle between the images before and after dye unloading to reveal active nerve terminals (alternatively a pre-stimulation image can be subtracted from a post-stimulation image). An ideal ROI size is one that is slightly bigger than a typical nerve terminal (Figure 3c).

7. Obtain the total/integrated fluorescence intensity of each ROI over time and export to Microsoft Excel (Figure 3d and 4a).

8. Normalise ROI traces to the same arbitrary value by aligning traces in the plane of the Y-axis for the first unloading stimulus in both $\mathrm{S} 1$ and $\mathrm{S} 2$ phases (Figure $4 \mathrm{~b}-\mathrm{c}$ ). This is to control for small variations in background fluorescence intensity.

9. Measure the absolute decrease in fluorescence evoked by each unloading stimulus in arbitrary fluorescence units for S1 and S2 as follows (Figure 4d):

- $\quad \mathrm{RRP}=$ Change in fluorescence $(\Delta \mathrm{F})$ triggered by $30 \mathrm{~Hz} 2 \mathrm{~s}$

- $\mathrm{RP}=$ Sum of $\Delta \mathrm{F}$ triggered by $3 \times 40 \mathrm{~Hz} 10 \mathrm{~s}$

- Total recycling pool $=\mathrm{RRP}+\mathrm{RP}$

10. For each relevant parameter in 7.9 , calculate the mean value over all nerve terminals of a single experiment.

11. For statistical analysis, mean values obtained from multiple independent experiments can be averaged. The number of coverslips rather than the number of nerve terminals should be used as the statistical $n$.

\section{Representative Results:}

A control experiment where CGNs underwent two rounds of identical loading and unloading steps is represented in Figure 5. When commencing a series of experiments, it is essential that a control experiment such as this is performed each day to confirm that S1 and S2 are comparable before varying experimental conditions during S2.

In this example, CGNs were loaded with $10 \mu \mathrm{M}$ FM1-43 using an $80 \mathrm{~Hz} 10 \mathrm{~s}$ stimulation (Figure 5a). Figure 5b shows FM1-43-loaded nerve terminals represented by fluorescent puncta. ROls were defined over 90 nerve terminals as shown in Figure $5 \mathrm{c}$. The same set of ROls was used for both S1 and S2. During both unloads, the RRP was first unloaded with a $30 \mathrm{~Hz}(2 \mathrm{~s})$ stimulation followed by RP unloading with 3 sequential $40 \mathrm{~Hz}(10 \mathrm{~s}$ ) stimuli (Figure $5 \mathrm{a}$ ). The fluorescence drop during each stimulus can be clearly observed and quantified (Figure $5 \mathrm{~d}-\mathrm{e}$ ). When examined, the fluorescence drops corresponding to the RRP, RP and total recycling pool were comparable in both S1 and S2. In addition, $20 \%$ of recycled SVs resided in the RRP while $80 \%$ resided in the RP in both S1 and S2.

A)
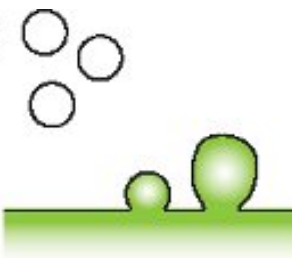

FM dye uptake (Load)
B)

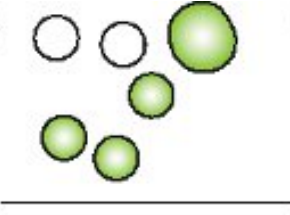

Washout
C)

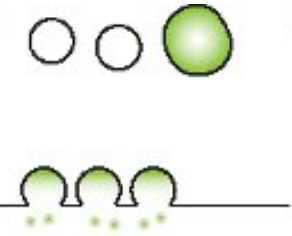

Release (Unload)

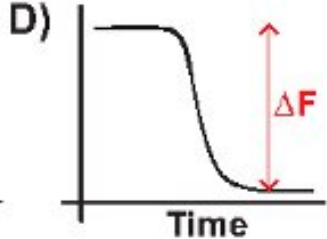

Quantification by

Figure 1 Schematic diagram of a typical FM experiment. A) SV endocytosis is triggered in the presence of FM dye (represented in green). The dye is taken up by invaginating membrane (single SVs or bulk endosomes). B) Non-internalised dye on the plasma membrane is washed 
away by perfusion. C) Upon application of an unloading stimulus, labeled SVs that have become available for release fuse with the plasma membrane resulting in a loss of fluorescence. D) The change in fluorescence $(\Delta \mathrm{F})$ which is proportional to the amount of released labeled SVs can then be quantified.

\section{A) Control}

\begin{tabular}{|c|c|c|c|c|c|c|c|c|}
\hline S1 Load & $10 \mathrm{mit}$ & \multicolumn{2}{|c|}{ S1 Unload } & \multirow{2}{*}{$\stackrel{20 \mathrm{~min}}{\longrightarrow}$} & \multirow{2}{*}{$\begin{array}{l}\text { S2 Load } \\
\text { FM dye }\end{array}$} & \multirow{2}{*}{$\stackrel{10 \mathrm{~min}}{\longrightarrow}$} & \multicolumn{2}{|c|}{ S2 Unload } \\
\hline FM dy & & RRP & $\mathrm{RP}$ & & & & RRP & RP \\
\hline Stimuli & & $30 \mathrm{~Hz} 2 \mathrm{~s}$ & $3 \times 40 \mathrm{~Hz} 10 \mathrm{~s}$ & & Stimuli & & $30 \mathrm{~Hz} 2 \mathrm{~s}$ & $3 \times 40 \mathrm{~Hz} 10 \mathrm{~s}$ \\
\hline
\end{tabular}

\section{B) Test for effect on endocytosis}

\begin{tabular}{|c|c|c|c|c|c|c|c|c|}
\hline S1 Load & $10 \mathrm{mi}$ & \multicolumn{2}{|c|}{ S1 Unload } & \multirow{2}{*}{$\stackrel{20 \mathrm{~min}}{\longrightarrow}$} & \multirow{2}{*}{$\begin{array}{l}\text { S2 Load } \\
\text { FM dye }\end{array}$} & \multirow{2}{*}{$\stackrel{10 \mathrm{~min}}{\longrightarrow}$} & \multicolumn{2}{|c|}{ S2 Unload } \\
\hline FM dy & & RRP & RP & & & & RRP & RP \\
\hline Stimuli & & $30 \mathrm{~Hz} 2 \mathrm{~s}$ & $3 \times 40 \mathrm{~Hz} 10 \mathrm{~s}$ & & Stimuli & & $30 \mathrm{~Hz} 2 \mathrm{~s}$ & $3 \times 40 \mathrm{~Hz} 10 \mathrm{~s}$ \\
\hline
\end{tabular}

\section{Test substance}

\section{C) Test for effect on exocytosis}

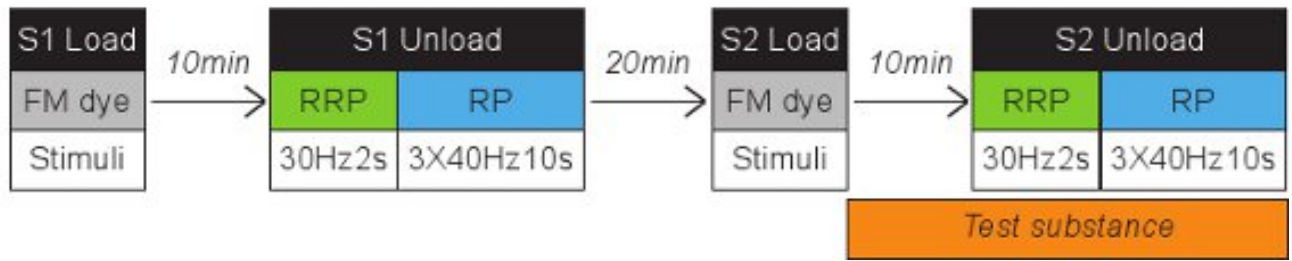

Figure 2 Schematic diagrams of possible experimental protocols. A) Flow chart of a control experiment where cells undergo two rounds of FM dye loading and unloading (S1 and S2). Cells can be loaded using a range of different stimuli. Unloading steps are identical in that the RRP is unloaded with $30 \mathrm{~Hz}$ for $2 \mathrm{~s}$ followed by RP unload using 3 times $40 \mathrm{~Hz}$ for $10 \mathrm{~s}$. RRP and reserve pool unloading stimuli were separated by 40 $\mathrm{sec}$, all other stimuli by $30 \mathrm{sec}$. Cells are left to recover for $20 \mathrm{~min}$ between S1 and S2. Flow charts of possible modifications to test the effect of a substance on either $\mathrm{B}$ ) endocytosis or $\mathrm{C}$ ) exocytosis are also shown. Corresponding test drug can be perfused into the chamber during indicated periods. 

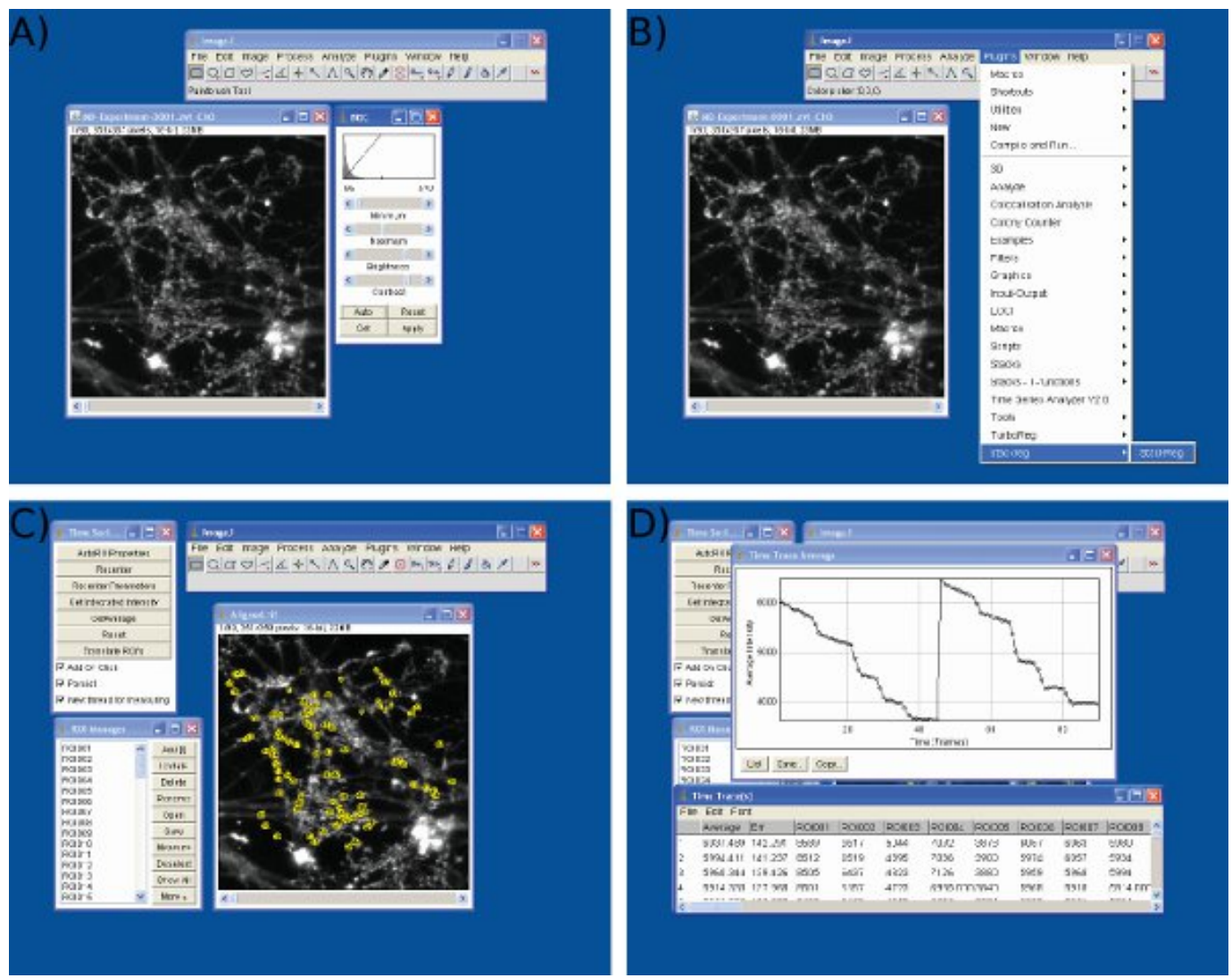

Figure 3 Screenshots of data analysis in Image J. Screenshots are shown for A) brightness and contrast adjustment, B) frame alignment, C) ROls selection, and D) intensity values extraction using Image $\mathrm{J}$.
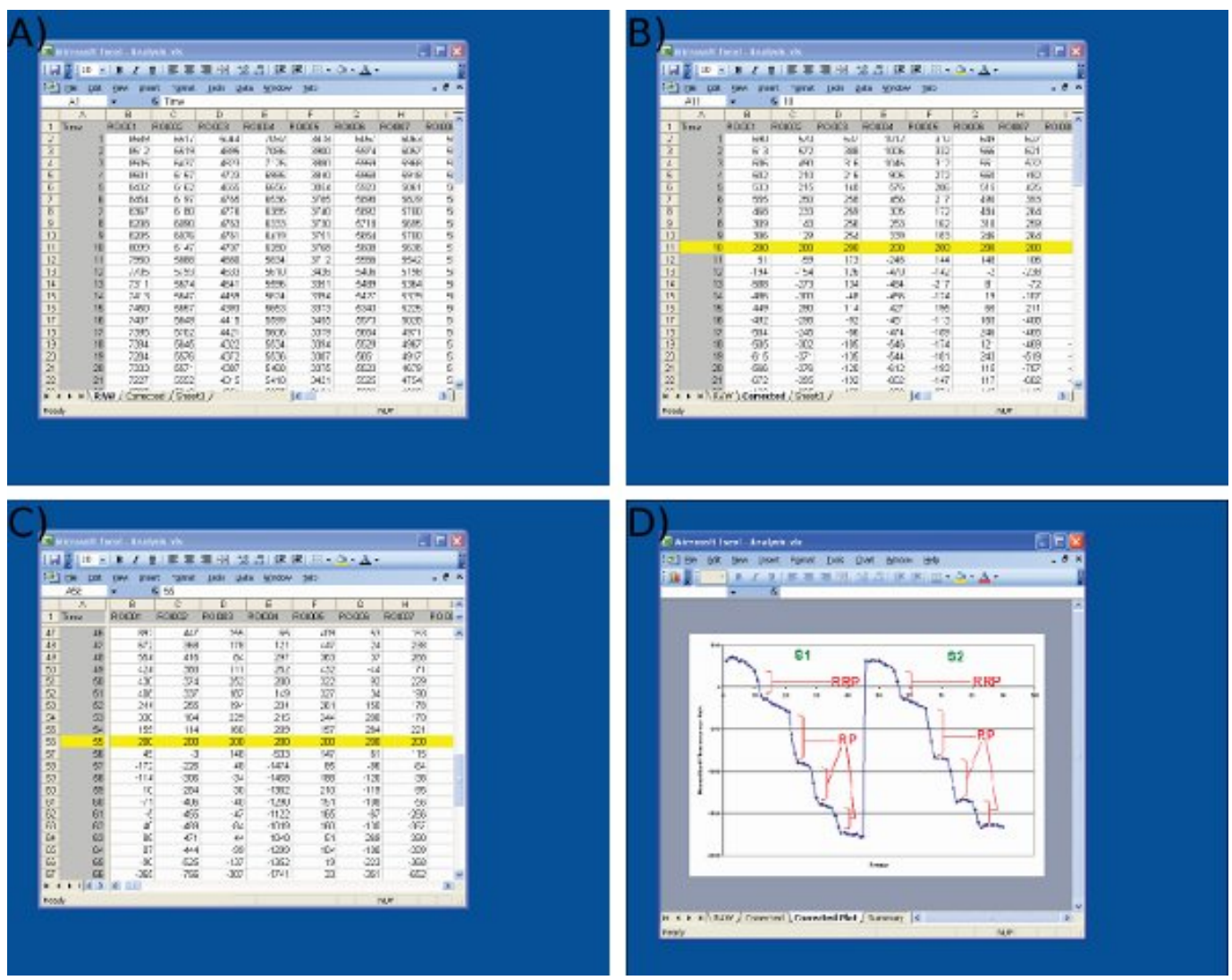
Figure 4 Screenshots of data analysis in Microsoft Excel. Screenshots are shown for A) importing raw data from Image $\mathrm{J}\left(1^{\text {st }}\right.$ column $=$ frame number, remaining columns $=$ data from individual nerve terminals) B) adjustment of $S 1$ baseline values (frame 10) to an arbitrary value (200) at the start of the first stimulus, C) adjustment of S2 baseline values at frame 55 using an identical protocol to S1, and D) measurement of fluorescence drops using Microsoft Excel. Note that the averaged trace shown in $D$ is used to define time points before and after each drop. The size of the fluorescence drops for each $\mathrm{ROI}$ should be determined from values on the spreadsheet shown in $\mathrm{C}$.
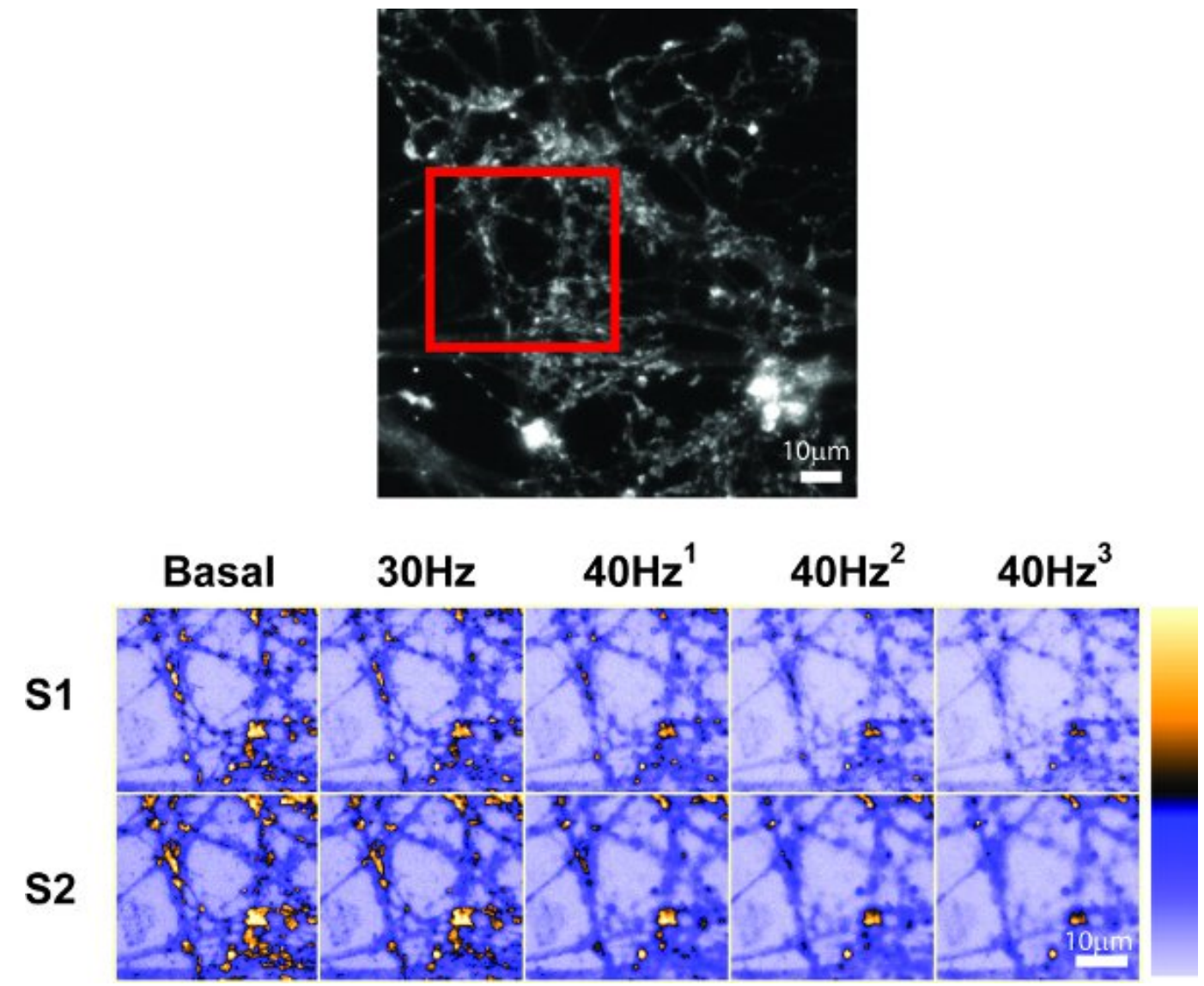

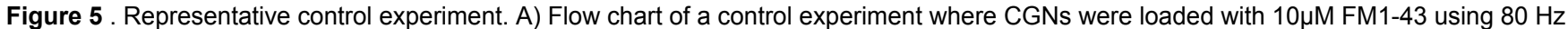
$(10 \mathrm{~s})$ stimulation. The S1 and S2 phases are identical. RRP and reserve pool unloading stimuli were separated by 40 sec, all other stimuli by 30 sec. B) An image showing nerve terminals loaded with FM1-43. C) The same image as B showing 90 numbered ROls selected for analysis. D) Images of an area depicted by a red box in B at selected time points. Basal = before stimulation; $30 \mathrm{~Hz}=$ after $30 \mathrm{~Hz} 2 \mathrm{~s}$ stimulation; $40 \mathrm{~Hz}{ }^{1,2,3}=$ after each $40 \mathrm{~Hz} 10 \mathrm{~s}$ stimulation. These images are presented in pseudocolor to illustrate changes in fluorescence (spectrum bar displayed on the right). E) Mean \pm SEM trace obtained from 90 nerve terminals depicted in C. Individual stimuli are represented by horizontal bars. Scale bars $=10 \mu \mathrm{m}$.

\section{Discussion}

FM dyes are extensively used to investigate nerve terminal function in many neuronal preparations. They have been employed mainly to monitor the extent of either SV endocytosis, SV turnover or the kinetics of exocytosis ${ }^{6}$. The described protocol extends these studies to examine the differential unloading of specific SV pools. This provides additional information regarding the replenishment of SV pools and also their extent of mobilisation.

FM dyes can be used to label multiple rounds of SV recycling within the same nerve terminals. We have exploited this property and designed protocols in which SV turnover in each terminal can be monitored twice in the same nerve terminals. This provides an accurate internal control, which is essential due to the heterogeneous nature of SV recycling in parallel nerve terminals ${ }^{11}$. Via the use of the S1 phase as an internal control, the refilling of the RRP, RP and the total SV pool in the presence of drugs can be reliably and directly compared.

In addition to providing information of the absolute size of the recycling, RRP and RP pools under different stimulation conditions, this protocol can also provide data for the following -1) The partitioning of SVs between the RRP and RP as a function of the recycling pool for S1 and S2, 2) the relative size of the S2 pools (RRP and RP) as a function of total S1 recycling pool and 3) the relative size of any defined SV pool in S2 as a function of the same pool in S1. This particular protocol will not provide information on unloading kinetics however, since the acquisition time is too slow (for kinetic measurements acquisition times should be as rapid as possible and unloading automatically synchronized to image capture).

Our $30 \mathrm{~Hz} 2 \mathrm{~s}$ stimuli evokes an identical extent of RRP unloading to hypertonic sucrose ${ }^{8}$. Since the size of the RRP is defined by hypertonic sucrose unloading ${ }^{15}$, we can state that this protocol unloads all RRP SVs, in agreement with studies in hippocampal neurons ${ }^{16}$. The reserve pool is almost completely depleted by three trains of 400 stimuli $(40 \mathrm{~Hz} 10 \mathrm{~s}$ each) since this stimulation unloads an identical amount of dye to 
a paradigm (2 stimuli with $50 \mathrm{mM} \mathrm{KCl}$ ) that depletes $95 \%$ of all dye-labelled SVs $\mathrm{s}^{8,17}$. Accurate quantification of the size of both the RRP and reserve pool is also dependent on acquiring information within the linear dynamic range of the CCD camera.

This simple protocol can also be modified further. The strength of loading stimuli can also be varied to determine how neuronal activity and different endocytosis modes affect SV pool replenishment. Furthermore, greater than two cycles of loading and unloading can also be performed if required. This protocol can also be used in cells transfected with either overexpression or shRNA vectors. Due to the low transfection efficiency of primary neuronal cultures, expressed proteins must be tagged with fluorescent proteins. It is essential that these fluorescent tags do not interfere with the FM dye signal (use cyan or red proteins, for example). In this instance, nerve terminals from transfected and non-transfected cells in the same field of view can also be compared as an additional control ${ }^{8}$. In such experiments a comparison of the extent of loading between S1 and S2 loads is of little value, since the perturbation is present during both loads. Partitioning of dye between SV pools can still be visualized however'.

Genetic reporters called pHluorins can also be employed to monitor SV exocytosis and endocytosis in primary neuronal culture. These probes use a $\mathrm{pH}$-sensitive green fluorescent protein to the $\mathrm{pH}$ environment of luminal domains of tagged SV proteins such as VAMP, synaptophysin and VGLUT $1^{18}$. When used in conjunction with vesicular ATPase inhibitors, pHluorins can report both the kinetics and extent of SV pool mobilization $^{19}$. The FM-dye based approach described here has some advantages over the pHluorin technique, Firstly, FM dyes provide information on which SV endocytosis mode replenishes the RRP and reserve pools ${ }^{8}$. Secondly specific SV pools can be labeled with FM dyes that have different spectral properties ${ }^{20}$ and finally there is no requirement for transfection. FM dyes cannot provide information on SV traffic between the resting and recycling SV pools however (in contrast to pHluorins ${ }^{19}$ ), since by definition SVs have to be loaded with dye during endocytosis to be visible. Thus both FM dyes and pHluorins have strengths and weaknesses and are most powerful when utilized in independent experiments to address the same question.

High quality images are essential for valid analysis and reproducible results. While horizontal drift can be easily corrected, experiments where there is a drift in the Z-axis cannot be recovered. For this reason, it is important to re-focus images before commencing the S1 and S2 unloads. In cases when a significant fluorescent decay has occurred, decay corrections may be applied (usually by subtracting a previously recorded trace from FM-loaded cells in the absence of stimulation). However, it is suggested that decay correction is only performed for graphical representation and not to be used for any quantitative analysis.

\section{Disclosures}

We have nothing to disclose.

\section{Acknowledgements}

This work was supported by a grant from the Wellcome Trust (Ref: 084277).

\section{References}

1. Rizzoli, S.O. \& Betz, W.J. Synaptic vesicle pools. Nat. Rev. Neurosci. 6, 57-69 (2005)

2. Sudhof, T.C. The synaptic vesicle cycle revisited. Neuron. 28, 317-320 (2000).

3. Evans, G.J. \& Cousin, M.A. Activity-dependent control of slow synaptic vesicle endocytosis by cyclin-dependent kinase 5. J. Neurosci. 27, 401-411 (2007).

4. Clayton, E.L., \& Cousin, M.A. Differential labelling of bulk endocytosis in nerve terminals by FM dyes. Neurochem. Int. 53, 51-55 (2008).

5. Clayton, E.L., Evans, G.J., \& Cousin, M.A. Bulk synaptic vesicle endocytosis is rapidly triggered during strong stimulation. J. Neurosci. 28, 6627-6632 (2008).

6. Cousin, M.A. Use of FM1-43 and other derivatives to investigate neuronal function. Curr. Protoc. Neurosci. Chapter 2, Unit 2, 6 (2008).

7. Clayton, E.L., et al. The phospho-dependent dynamin-syndapin interaction triggers activity-dependent bulk endocytosis of synaptic vesicles. J. Neurosci. 29, 7706-7717 (2009).

8. Cheung, G., Jupp, O.J., \& Cousin, M.A. Activity-dependent bulk endocytosis and clathrin-dependent endocytosis replenish specific synaptic vesicle pools in central nerve terminals. J. Neurosci. 30, 8151-8161 (2010).

9. Betz, W.J., Mao, F., \& Smith, C.B. Imaging exocytosis and endocytosis. Curr. Opin. Neurobiol. 6, 365-371 (1996).

10. Ryan, T.A., Reuter, H., \& Smith, S.J. Optical detection of a quantal presynaptic membrane turnover. Nature. 388, 478-482 (1997).

11. Murthy, V.N., Sejnowski, T.J., \& Stevens, C.F. Heterogeneous release properties of visualized individual hippocampal synapses. Neuron. 18, 599-612 (1997).

12. Tan, T.C., et al. Cdk5 is essential for synaptic vesicle endocytosis. Nat. Cell. Biol. 5, 701-710 (2003).

13. Anggono, V., Cousin, M.A., \& Robinson P.J. Styryl dye-based synaptic vesicle recycling assay in cultured cerebellar granule neurons. Methods. Mol. Biol. 457, 333-345 (2008).

14. Gallo, V., et al. Selective release of glutamate from cerebellar granule cells differentiating in culture. Proc. Natl. Acad. Sci. U.S.A. 79, 7919-7923 (1982).

15. Stevens, C.F. Neurotransmitter release at central synapses. Neuron. 40, 381-388 (2003).

16. Mozhayeva, M.G., et al. Development of vesicle pools during maturation of hippocampal synapses. J. Neurosci. 22, 654-665 (2002).

17. Cousin, M.A., \& Evans, G.J.O. Activation of silent and weak synapses by cAMP-dependent protein kinase in cultured cerebellar granule neurons. J. Physiol. 589, 1943-1955 (2011).

18. Kim, S.H., \& Ryan, T.A. Synaptic vesicle recycling at CNS synapses without AP-2. J. Neurosci. 29, 3865-3874 (2009).

19. Kim, S.H., \& Ryan, T.A. Cdk5 serves as a major control point in neurotransmitter release. Neuron. 67, 797-809 (2010).

20. Groemer, T.W. \&, Klingauf, J. Synaptic vesicles recycling spontaneously and during activity belong to the same pool. Nat. Neurosci. 10, 145-147 (2007). 